\title{
Thermodiffusion im kritischen Verdampfungsgebiet binärer Systeme
}

\author{
R. HaAse, H.-W. BorgmanN, K.-H. DüCker und W.-P. LeE
}

Lehrstuhl für Physikalische Chemie II der Rheinisch-Westfälischen Technischen Hochschule Aachen

(Z. Naturforsch. 26 a, 1224-1227 [1971] ; eingegangen am 8. Mai 1971)

\begin{abstract}
Es werden Messungen des Thermodiffusionsfaktors für das fluide System Methan+Propan beschrieben und diskutiert. Dabei liegt der Druck zwischen 40 und 75 bar, die mittlere Temperatur bei rund $73^{\circ} \mathrm{C}$, der mittlere Molenbruch des Methans zwischen 0,23 und 0,63. Das System befindet sich in der Nähe eines kritischen Verdampfungspunktes, und zwar teils im Gaszustand, teils im hyperkritischen Gebiet. Die Prüfung eines auf Plausibilitätsbetrachtungen beruhenden Ansatzes für den Thermodiffussionsfaktor bei fluiden Zweistoffsystemen (Haase 1950) führt zu einem qualitativ befriedigenden Bild. Insbesondere ist die Aussage, daß der Thermodiffusionsfaktor bei Annäherung an einen kritischen Punkt unendlich wird, mit den Messungen am vorliegenden System und auch an anderen binären fluiden Systemen verträglich.
\end{abstract}

Nach unseren Untersuchungen über Diffusion ${ }^{1}$ und Thermodiffusion ${ }^{2}$ im kritischen Entmischungsgebiet binärer flüssiger Systeme wollen wir uns in der vorliegenden Arbeit mit der Thermodiffusion im kritischen Verdampfungsgebiet binärer Systeme beschäftigen.

Schon RUTHERFord und ROOF ${ }^{3}$ bestimmten experimentell Thermodiffusionsfaktoren im kritischen Verdampfungsgebiet des Systems Methan + n-Butan, wobei sie sich der kritischen Kurve vom flüssigen Zustand her näherten. Im Anschluß daran zeigte RUTHERFORD ${ }^{4}$, daß ein auf Plausibilitätsbetrachtungen beruhender Ansatz ${ }^{5,6}$ die Versuchsdaten recht gut beschreibt. Wir werden über entsprechende Messungen und Auswertungen am System Methan + Propan berichten, wobei wir die Umgebung der kritischen Kurve vom Gaszustand aus erreichen.

\section{Messungen}

Die Messungen der Thermodiffusion im System Methan + Propan beruhen auf der Ermittlung der stationären Zusammensetzungen der in zwei Kammern befindlichen Mischungen, wobei die unter gleichem Druck stehenden Kammern verschieden temperiert und voneinander durch eine Glasmembran getrennt sind ${ }^{7}$. Jede der beiden Kammern stellt einen Hohlzylinder aus Edelstahl (äußere Länge: 31,5 mm, äußerer Durchmesser: $40 \mathrm{~mm}$, inneres Volumen: $32,5 \mathrm{~cm}^{3}$ ) dar und ist

Sonderdruckanforderungen an Prof. Dr. R. HAASE, Lehrstuhl für Physikal. Chemie II der Rhein.-Westf. Techn. Hochschule, D-5100 Aachen, Templergraben 59.

1 R. Haase u. M. Siry, Z. Phys. Chem. Frankfurt 57, 56 [1968].

2 R. Haase u. B. K. Bienert, Ber. Bunsenges. Physik. Chem. 71, 392 [1967].

3 W. M. Rutherford u. J. G. Roof, J. Phys. Chem. 63, 1506 [1959]. von einem Temperiermantel aus Messing umgeben. Jeder Temperiermantel wird von einem Umlaufthermostaten mit Wasser durchspült. Durch Einbau einer elektrischen Zusatzheizung und eines Flüssigkeitskühlers wird erreicht, daß der Temperaturabfall auf die Membran beschränkt bleibt. Durch Hubrührer im Inneren der Kammern wird für gleichmäßige Durchmischung gesorgt. Weiterhin ist durch Vorversuche an Glasmembranen verschiedener Porosität sichergestellt, $\mathrm{da} \beta$ in den Poren der gewählten Membran tatsächlich nur Diffusion und Thermodiffusion auftreten, also Membraneffekte wie Thermoosmose keine Rolle spielen.

Da unsere Versuche einen Druckbereich von 40 bar bis 75 bar bei einer mittleren Temperatur von etwa $73{ }^{\circ} \mathrm{C}$ umfassen, sind der Gesamtaufbau der Apparatur, die Temperatur- und Druckmessungen, die Einfüllung der gasförmigen Substanzen und die Probeentnahme recht kompliziert. Die Einzelheiten müssen daher an anderer Stelle ${ }^{\boldsymbol{9}}$ nachgelesen werden. Hier sei nur erwähnt, daß man zur Erreichung des stationären Endzustandes rund 3 bis 4 Tage benötigt und die Analyse der Gemische gaschromatographisch erfolgt.

\section{Auswertung}

Der stationäre Zustand in einem binären nichtisothermen System gleichförmigen Druckes kommt durch das Gegeneinanderwirken von Diffusion und Thermodiffusion zustande. Das System besteht in unserem Falle aus dem Inneren der Glasmembran mit den beiden Kammern als Stoffbehältern, die je-

4 W. M. Rutherford, A. I. Ch. E. J. 9, 841 [1963].

5 R. HAase, Z. Physik 127, 1 [1950].

6 R. HAase, Z. Phys. Chem. 196, 219 [1950].

7 Frühere Versuche ${ }^{3,8}$ mit Sintermetallplatten führten zu Schwierigkeiten infolge der zu hohen Wärmeleitfähigkeit des Metalls.

8 H.-W. Borgmann, Dissertation, Aachen 1966.

9 WEN-Po LeE, Dissertation, Aachen 1971. 
weils gleichförmige Zusammensetzung aufweisen. Bei vorgegebenen Temperaturen an den Grenzen des Systems - hier: an den Membranoberflächen ist der stationäre Zustand durch eine zeitlich konstante Konzentrationsverteilung gekennzeichnet.

Wir wählen als unabhängige Variable zur Beschreibung des Zustandes eines Volumenelementes unseres Systems die thermodynamische Temperatur $T$, den Druck $P$ und den Molenbruch $x_{2}$ der Komponente 2. Liegen für $P=$ const die Gradienten von $T$ und $x_{2}$ nur in Richtung einer Raumkoordinate, wie dies bei unseren Versuchen der Fall ist, so gilt für den stationären Zustand ${ }^{10}$ :

$$
\mathrm{d} x_{2} / x_{1} x_{2}=\alpha_{T} \mathrm{~d} T / T .
$$

Hierin bezeichnet $x_{1}\left(=1-x_{2}\right)$ den Molenbruch der Komponente 1 und $\alpha_{T}$ den Thermodiffusionsfaktor, der so definiert ist, daß $\alpha_{T}>0$ Anreicherung der Komponente 2 in den Gebieten höherer Temperatur bedeutet. Die dimensionslose Größe $\alpha_{T}$ ist eine Funktion von $T, P$ und $x_{2}$.

Zur Ermittlung von $\alpha_{T}$ aus den Versuchsdaten müssen wir Gl. (1) zwischen den Grenzen $T^{\prime}, x_{2}{ }^{\prime}$ (erste Kammer) und $T^{\prime \prime}, x_{2}^{\prime \prime}$ (zweite Kammer) integrieren. Dabei soll $T^{\prime \prime}>T^{\prime}$ sein. Das Problem ist einfach, wenn die Differenz $\Delta T=T^{\prime \prime}-T^{\prime}(>0)$ so klein wie möglich gehalten wird. Dann nämlich kann $\alpha_{T}$ als Konstante bei der Integration angesehen werden. Mit $\alpha_{T}=$ const und $x_{1}+x_{2}=1$ erhalten wir aus Gl. (1) :

mit

$$
\ln \left(r^{\prime \prime} / r^{\prime}\right)=\alpha_{T} \ln \left(T^{\prime \prime} / T^{\prime}\right)
$$

$$
r^{\prime} \equiv x_{2}{ }^{\prime} / x_{1}{ }^{\prime}=n_{2}{ }^{\prime} / n_{1}{ }^{\prime}, \quad r^{\prime \prime} \equiv x_{2}^{\prime \prime} / x_{1}{ }^{\prime \prime}=n_{2}^{\prime \prime} / n_{1}{ }^{\prime \prime} \text {. }
$$

Hierbei ist $n_{i}^{\prime}$ bzw. $n_{i}^{\prime \prime}$ die Stoffmenge der Komponente $i$ in der ersten bzw. zweiten Kammer. Der Thermodiffusionsfaktor $\alpha_{T}$ in Gl. (2) bezieht sich auf den vorgegebenen Druck $P$, die mittlere Temperatur $\langle T\rangle=\left(T^{\prime}+T^{\prime \prime}\right) / 2$ und den mittleren Molenbruch $\left\langle x_{2}\right\rangle=\left(x_{2}{ }^{\prime}+x_{2}^{\prime \prime}\right) / 2$.

Variiert man bei festen Werten von $P,\langle T\rangle$ und $\left\langle x_{2}\right\rangle$ die Temperaturdifferenz $\Delta T$, so muß $\alpha_{T}$, berechnet nach Gl. (2), konstant bleiben, falls $\Delta T$ hinreichend klein ist und somit $\mathrm{Gl}$. (2) überhaupt gilt. Entsprechende Experimente ${ }^{9}$ zeigen, daß Gl. (2) unter der Bedingung $\Delta T \leqq 8 \mathrm{~K}$ anwendbar ist. Wir wählen daher für unsere Versuche $\Delta T$ in der Nähe von $8 \mathrm{~K}$.

10 Vgl. R. HaAse, Thermodynamik der irreversiblen Prozesse, Verlag Steinkopff, Darmstadt 1963.
Bei den folgenden Ausführungen über das System Methan + Propan soll Propan als Komponente 1 und Methan als Komponente 2 betrachtet werden. Dann bedeutet die Aussage $\alpha_{T}>0$ Anreicherung der "leichteren" Komponente (des Methans) an der „wärmeren" Stelle. Auch schreiben wir einfachheitshalber $x_{2}$ für den mittleren Molenbruch $\left\langle x_{2}\right\rangle$ des Methans (als Maß für die mittlere Zusammensetzung) und $T$ für die mittlere Temperatur $\langle T\rangle$, die hier stets $346,08 \mathrm{~K}$ (rund $73^{\circ} \mathrm{C}$ ) beträgt.

\section{Ergebnisse}

Der Druck und die mittlere Zusammensetzung sind bei unseren Versuchen stets so gewählt worden, daß man einerseits nicht das Zweiphasengebiet (Gebiet des Verdampfungsgleichgewichtes) erreicht, sich aber andererseits von der Seite der Gasphase her so weit wie möglich dem kritischen Punkt für das Verdampfungsgleichgewicht nähert. Dabei gelangt man teilweise in das hyperkritische Gebiet. Der erwähnte kritische Punkt liegt bei Darstellung im $T$-P- $x_{2}$-Zustandsraum auf einer Raumkurve, der kritischen Verdampfungskurve, die für das System Propan + Methan experimentell bekannt ist ${ }^{11}$.

In Abb. 1 ist das isotherme Verdampfungsgleichgewicht (Dampfdruck als Funktion der Zusammensetzung der flüssigen Phase bzw. der Gasphase) für das System Propan + Methan bei 346,08 K graphisch dargestellt, wobei auch die Lage des kritischen Punktes ersichtlich ist. Weiterhin sind die Drucke und (mittleren) Zusammensetzungen, bei denen die Thermodiffusionsfaktoren ermittelt wurden, eingezeichnet. Die Dampfdruckkurve in Abb. 1 ist mit Hilfe einer empirischen Zustandsgleichung (siehe unten) konstruiert worden, weil im interessierenden Zustandsgebiet keine direkten Meßdaten vorliegen.

In Tab. 1 finden sich die experimentellen Werte $\left[\alpha_{T}(\exp )\right]$ des Thermodiffusionsfaktors, die nach dem eingangs geschilderten Verfahren aus den Meßdaten mit Hilfe von Gl. (2) für das in Abb. 1 gekennzeichnete Zustandsgebiet bestimmt worden sind.

Die Messungen der Temperatur bzw. des Molenbruches des Methans sind mit einem Fehler von $\pm 0,1 \mathrm{~K}$ bzw. $\pm 0,005$ behaftet. Damit ergibt sich nach dem Gaußschen Gesetz der Fehlerfortpflanzung

11 Vgl. J. G. Roof u. J. D. Baron, J. Chem. Eng. Data 12, 292 [1967]. 


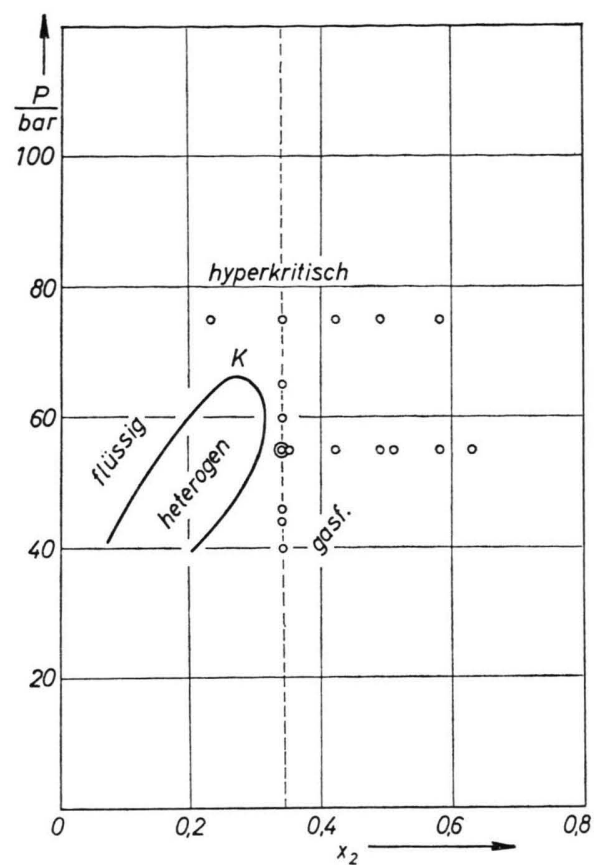

Abb. 1. System Propan+Methan bei 346,08 K: Dampfdruck $P$ in Abhängigkeit vom Molenbruch $x_{2}$ des Methans in der flüssigen Phase bzw. in der Gasphase (K: kritischer Punkt mit den Koordinaten $P=P_{\mathrm{K}}=66,4$ bar, $\left.x_{2}=x_{\mathrm{K}}=0,28\right)$; $\circ \circ$ Lage der Meßpunkte bei den Thermodiffusionsversuchen.

Tab. 1. System Propan +Methan bei einer mittleren Temperatur von 346,08 K: Experimentelle Werte $\left[\alpha_{\Gamma}(\exp )\right]$ und nach Gl. (4) berechnete Werte $\left[\alpha_{T}\right.$ (ber)] des Thermodiffusionsfaktors $\alpha_{T}$ in Abhängigkeit vom Druck $P$ und vom mittleren Molenbruch $x_{2}$ des Methans mit Angabe der Größe $\alpha_{T}{ }^{0}$ [siehe Gl. (5)].

\begin{tabular}{lllrr}
\hline$P /$ bar & $x_{2}$ & $\alpha_{T}^{0}$ & $\alpha_{T}(\exp )$ & $\alpha_{T}$ (ber) \\
\hline 40 & 0,34 & 0,1004 & 0,79 & 2,50 \\
44 & 0,34 & 0,1004 & 2,01 & 3,42 \\
46 & 0,34 & 0,1004 & 2,88 & 4,05 \\
55 & 0,34 & 0,1004 & 12,51 & 10,01 \\
& 0,35 & 0,1008 & 12,48 & 9,58 \\
& 0,42 & 0,1054 & 2,67 & 4,42 \\
& 0,49 & 0,1102 & 1,30 & 2,94 \\
& 0,51 & 0,1116 & 0,70 & 2,62 \\
& 0,58 & 0,1167 & 0,36 & 2,01 \\
60 & 0,63 & 0,1212 & 0,47 & 1,71 \\
65 & 0,34 & 0,1004 & 34,24 & 19,82 \\
75 & 0,34 & 0,1004 & 18,26 & 37,18 \\
& 0,23 & 0,0944 & 15,51 & 10,55 \\
& 0,34 & 0,1004 & 8,96 & 42,80 \\
& 0,42 & 0,1054 & 4,59 & 15,42 \\
& 0,49 & 0,1102 & 2,46 & 7,49 \\
& 0,58 & 0,1167 & 1,83 & 3,96 \\
\hline
\end{tabular}

bei Beachtung von Gl. (2) und (3) für den Thermodiffusionsfaktor ein zufälliger Fehler von $\pm 0,006$. Es ist allerdings bei den komplizierten Meßoperationen mil bedeutend höheren systematischen Fehlern zu rechnen.

\section{Prüfung eines Ansatzes}

Plausibilitätsbetrachtungen im Rahmen der Thermodynamik der irreversiblen Prozesse ${ }^{10}$ legen folgenden Ansatz ${ }^{5,6}$ für den Thermodiffusionsfaktor $\alpha_{T}$ bei binären fluiden Systemen nahe:

mit

$$
\begin{gathered}
\alpha_{T}=\frac{\alpha_{T}{ }^{0}\langle M\rangle R T+M_{1} h_{2}-M_{2} h_{1}}{\langle M\rangle \psi} \\
a_{T}{ }^{0} \equiv \lim _{P \rightarrow 0} \alpha_{T}, \\
\langle M\rangle \equiv M_{1} x_{1}+M_{2} x_{2}, \\
h_{i} \equiv H_{i}-H_{i}^{0} \quad(i=1,2), \\
H_{i}{ }^{0} \equiv \lim _{P \rightarrow 0} H_{i} \quad(i=1,2), \\
\psi \equiv x_{1}\left(\partial \mu_{1} / \partial x_{1}\right)_{T, P}=x_{2}\left(\partial \mu_{2} / \partial x_{2}\right)_{T, P} .
\end{gathered}
$$

Hierin bedeutet $R$ die Gaskonstante, $M_{i}$ die molare Masse, $H_{i}$ die partielle molare Enthalpie und $\mu_{i}$ das chemische Potential der Komponente $i(i=1,2)$.

Die Größe $\alpha_{T}^{0}$, die nur von $T$ und $x_{2}$ abhängt, ist der Grenzwert des Thermodiffusionsfaktors bei verschwindendem Druck und läßt sich entweder gemäß Gl. (5) aus der Extrapolation von Meßwerten der Funktion $\alpha_{T}(P)$ oder aus der Molekulartheorie gewinnen. Überhaupt ist zur Zeit die quantitative molekularkinetische Berechnung des Thermodiffusionsfaktors allein für den idealen Gaszustand, also lediglich für die Größe $\alpha_{T}^{0}$ möglich.

Die Funktionen $h_{1}, h_{2}$ und $\psi$ sind aus der $\mathrm{Zu}$ standsgleichung der binären fluiden Mischung ermittelbar. Es gelten die Bedingungen

$$
\lim _{P \rightarrow 0} h_{i}=0 \quad(i=1,2), \quad \lim _{P \rightarrow 0} \psi=R T .
$$

Dies folgt aus Gl. (7) - (9) und der bekannten Beziehung

$$
\mu_{i} \rightarrow \mu_{i}^{*}+R T \ln \left(P x_{i} / P^{\dagger}\right) \text { für } P \rightarrow 0 \quad(i=1,2),
$$
worin $\mu_{i}^{*}$ eine Temperaturfunktion und $P^{\dagger}$ einen Standarddruck darstellt.

Obwohl Gl. (4) nur den ,thermodynamischen Anteil" der Druckabhängigkeit des Thermodiffusionsfaktors wiedergibt ${ }^{6}$, hat sich dieser Ansatz, der keine freien Parameter enthält, erstaunlich gut bewährt ${ }^{4-6}$. Wir wollen daher Gl. (4) auch an den vorliegenden Meßdaten prüfen.

Die Größe $\alpha_{T}{ }^{0}$ wird nach der ersten Näherung der molekularkinetischen Theorie von CHAPMAN und ENSKOG berechnet ${ }^{12}$. Dabei wird das (6.12)-Modell von LENNARD-JonEs zugrunde gelegt. Mit Hilfe der in der Literatur ${ }^{13}$ angegebenen Stoßintegrale und Molekülparameter lassen sich so die numerischen Werte von $\alpha_{T}{ }^{0}$ ermitteln ${ }^{8,9}$. Diese Werte sind in Tab. 1 aufgeführt. Wie man erkennt, stellt $\alpha_{T}{ }^{0}$ fast 
stets nur einen kleinen Bruchteil des gemessenen Thermodiffusionsfaktors dar.

Die Größen $h_{1}, h_{2}$ und $\psi$ werden aus den thermodynamischen Funktionen gewonnen ${ }^{9}$, die BENEDICT, WEBB und RUBIN ${ }^{14}$ für binäre fluide Mischungen aus Kohlenwasserstoffen angegeben haben und die einer Zustandsgleichung mit 8 empirischen Parametern für jede Komponente entsprechen. Wir haben uns davon überzeugt, daß die so berechneten thermodynamischen Größen das Phasenverhalten des Systems Propan + Methan (vgl. Abb. 1) nahezu quantitativ wiedergeben ${ }^{15}$. Die in Gl. (4) benutzten Werte für $h_{1}, h_{2}$ und $\psi$ sind also mit dem empirisch bekannten Gleichgewichtsverhalten des Systems verträglich.

Die auf diese Weise nach Gl. (4) berechneten Werte des Thermodiffusionsfaktors sind in Tab. 1 unter der Bezeichnung $\alpha_{T}$ (ber) aufgeführt.

In Abb. 2 und Abb. 3 werden gemessene und be. rechnete Werte des Thermodiffusionsfaktors einander nochmals gegenübergestellt.
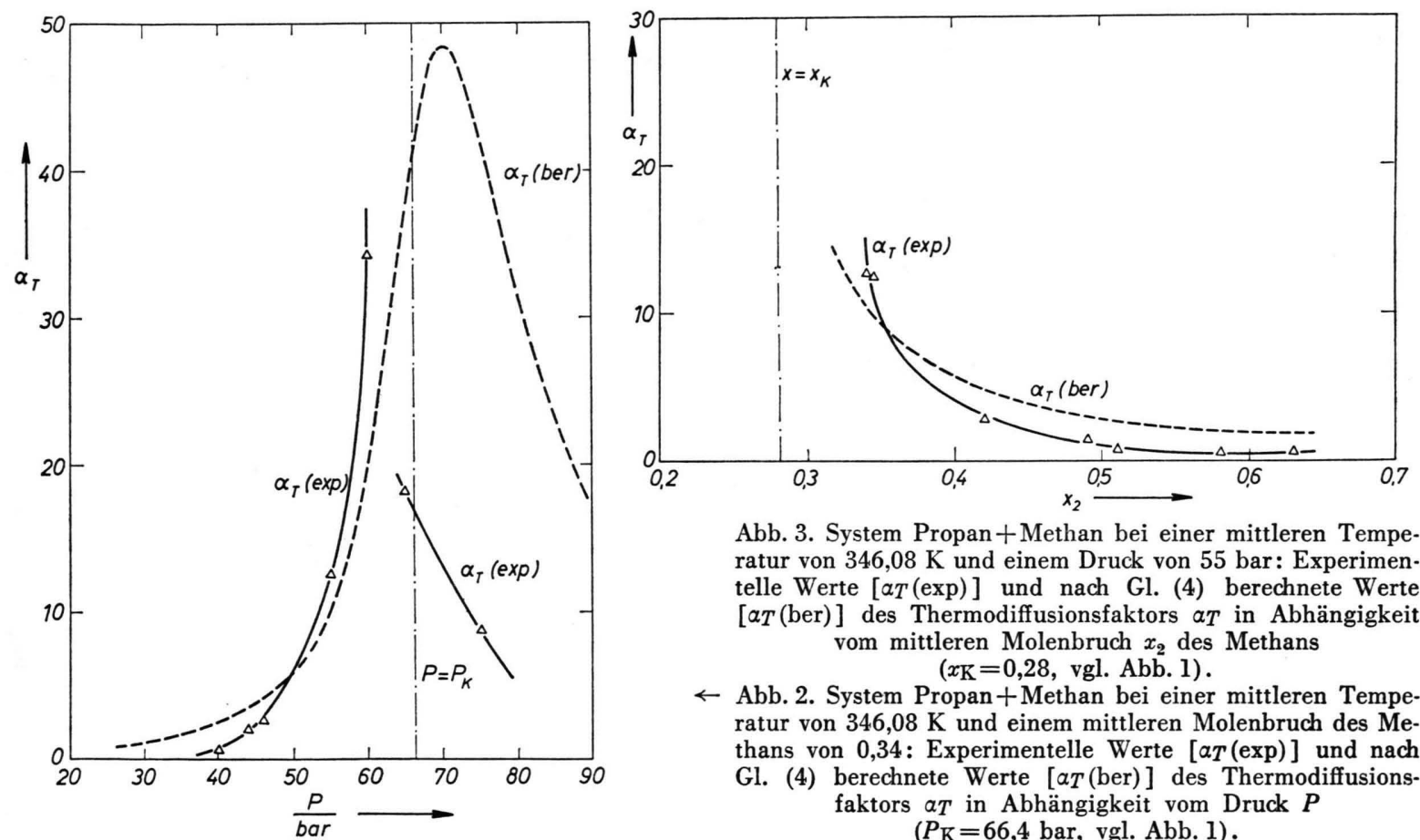

Abb. 3. System Propan + Methan bei einer mittleren Temperatur von 346,08 $\mathrm{K}$ und einem Druck von 55 bar: Experimentelle Werte $\left[\alpha_{T}(\exp )\right]$ und nach Gl. (4) berechnete Werte $\left[\alpha_{T}\right.$ (ber)] des Thermodiffusionsfaktors $\alpha_{T}$ in Abhängigkeit vom mittleren Molenbruch $x_{2}$ des Methans $\left(x_{\mathrm{K}}=0,28\right.$, vgl. Abb. 1$)$.

$\leftarrow$ Abb. 2. System Propan+Methan bei einer mittleren Temperatur von $346,08 \mathrm{~K}$ und einem mittleren Molenbruch des Methans von 0,34: Experimentelle Werte $\left[\alpha_{T}(\exp )\right]$ und nach Gl. (4) berechnete Werte $\left[a_{T}\right.$ (ber)] des Thermodiffusionsfaktors $\alpha_{T}$ in Abhängigkeit vom Druck $P$ $\left(P_{\mathrm{K}}=66,4\right.$ bar, vgl. Abb. 1$)$.

12 S. Chapman u. T. G. Cowling, The Mathematical Theory of Non-Uniform Gases, Third Edition, Cambridge University Press, Cambridge 1970.

13 J. O. Hirschfelder, R. B. Bird u. E. L. Spotz, J. Chem. Phys. 16, 968 [1948].

14 M. Benedict, G. B. Webb u. L. C. Rubin, J. Chem. Phys. 10, 747 [1942].
Wie man erkennt, beschreibt der Ansatz (4) das Verhalten des Thermodiffusionsfaktors beim System Propan + Methan qualitativ richtig. Die in quantitativer Hinsicht teilweise erheblichen Abweichungen sind durch die unterschiedlichen Lagen der Maxima in den gemessenen und berechneten Funktionen $\alpha_{T}(P)$ bzw. $\alpha_{T}\left(x_{2}\right)$ bedingt. Diese Maxima, die in der Nähe des kritischen Punktes liegen, sind sehr empfindlich gegenüber kleinen Meßungenauigkeiten dynamischen Bedingung

gelten:

$$
\psi=0 \quad \text { (kritische Kurve) }
$$

Die Aussage (12) ist mit den Messungen verträgGl. (12) ist auch für das kritische Verdampfungsgebiet des Systems n-Butan +Methan ${ }^{3,4}$ und für das kritische Entmischungsgebiet flüssiger Zweistoffsysteme ${ }^{2}$ bestätigt worden. Unsicherheiten in der Zustandsgleichung. 